\title{
A needs-based methodology to project physicians and nurses to 2030: the case of the Kingdom of Saudi Arabia
}

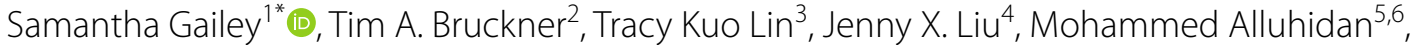 \\ Taghred Alghaith ${ }^{5}$, Hussah Alghodaier ${ }^{5}$, Nabiha Tashkandi ${ }^{7}$, Christopher H. Herbst ${ }^{8}$, Mariam M. Hamza ${ }^{8}$ and \\ Nahar Alazemi ${ }^{5}$
}

\begin{abstract}
Background: The Kingdom of Saudi Arabia (KSA), as part of its 2030 National Transformation Program, set a goal of transforming the healthcare sector to increase access to, and improve the quality and efficiency of, health services. To assist with the workforce planning component, we projected the needed number of physicians and nurses into 2030. We developed a new needs-based methodology since previous global benchmarks of health worker concentration may not apply to the KSA.

Methods: We constructed an epidemiologic "needs-based" model that takes into account the health needs of the KSA population, cost-effective treatment service delivery models, and worker productivity. This model relied heavily on up-to-date epidemiologic and workforce surveys in the KSA. We used demographic population projections to estimate the number of nurses and physicians needed to provide this core set of services into 2030 . We also assessed several alternative scenarios and policy decisions related to scaling, task-shifting, and enhanced public health campaigns.

Results: When projected to 2030, the baseline needs-based estimate is approximately 75,000 workers (5788 physicians and 69,399 nurses). This workforce equates to 2.05 physicians and nurses per 1000 population. Alternative models based on different scenarios and policy decisions indicate that the actual needs for physicians and nurses may range from 1.64 to 3.05 per 1000 population in 2030.

Conclusions: Based on our projections, the KSA will not face a needs-based health worker shortage in 2030. However, alternative model projections raise important policy and planning issues regarding various strategies the KSA may pursue in improving quality and efficiency of the existing workforce. More broadly, where country-level data are available, our needs-based strategy can serve as a useful step-by-step workforce planning tool to complement more economic demand-based workforce projections.
\end{abstract}

Keywords: Workforce planning, Needs based, Epidemiologic model, Kingdom of Saudi Arabia, Human resources for health

${ }^{*}$ Correspondence: sgailey@uci.edu

${ }^{1}$ School of Social Ecology, University of California Irvine, Irvine, CA, USA

Full list of author information is available at the end of the article

\section{Background}

Human resources play a crucial role in delivering health services. From a policy- and country-level perspective, health planners and decision-makers must ensure that the right number of people, with the right skills, deliver appropriate health services for the population's health original author(s) and the source, provide a link to the Creative Commons licence, and indicate if changes were made. The images or other third party material in this article are included in the article's Creative Commons licence, unless indicated otherwise in a credit line to the material. If material is not included in the article's Creative Commons licence and your intended use is not permitted by statutory regulation or exceeds the permitted use, you will need to obtain permission directly from the copyright holder. To view a copy of this licence, visit http://creativecommons.org/licenses/by/4.0/. The Creative Commons Public Domain Dedication waiver (http://creativeco mmons.org/publicdomain/zero/1.0/) applies to the data made available in this article, unless otherwise stated in a credit line to the data. 
needs. Given the substantial training and resources required to develop a health workforce, planners that can anticipate health workforce needs into the future will be better equipped to match these resources to the particular demographic and health characteristics of the population.

From a planning perspective, a foundational component to understanding the overall workforce that is needed requires an estimation of the health conditions of the population. Whereas several strategies have been proposed to arrive at estimates of an ideal volume and skill mix of the health workforce, most begin with trying to understand the population's morbidities that are amenable to cost-effective health care treatments and interventions. Some countries, for instance, have undergone population ageing, which increases the median age of the population and shifts the age structure into older ages. This ageing process reflects both the current age structure of the population (i.e., baby boomers reaching older ages) and lower fertility rates. As a result, "ageing" countries show an increasing predominance of chronic diseases as the leading causes of disability and death. Other populations may have a greater prevalence of risky behaviors (e.g., smoking) that produce a unique set of associated illnesses. Regardless of a country's specific demographic and health behavior profile, planning for an efficient health workforce should involve matching the health workforce to the distribution of health conditions in that population that health workers will address.

Starting in 2016, The Kingdom of Saudi Arabia (KSA) developed the ambitious National Transformation Program designed to achieve key objectives by 2030 which focus on 8 themes. Transformation of the healthcare sector in this high-income country serves as the first theme of the "Vision 2030" strategic framework. A core element of this transformation in the KSA involves increasing access to, and improving the quality and efficiency of, health services [1].

The fragmented nature of health subsystems in the KSA poses challenges to long-term strategic workforce planning. Although health care is currently provided free of charge to all Saudi citizens, the KSA is in the process of restructuring their healthcare system to privatize some health services for citizens and non-citizens [2]. Health services are delivered through hospitals and outpatient facilities operated by multiple public sector subsystems and, increasingly, the private sector. These facilities include approximately 500 hospitals (nearly one-third of which are operated by the private sector), 2000 public primary health centers, and 3000 private clinics and polyclinics. Specialized health centers also provide a range of health services, including dental, cardiology, and oncology [3].
Over the past decade, the health workforce in the KSA has grown steadily across all sectors. From 2011 to 2019 , the total health workforce increased from 303,578 to 467,650 . In 2019 , nurses comprised $43 \%(199,013)$ of all staff, allied health workers (including applied medical specialists and technicians) $26 \%(123,619)$, physicians $20 \%(94,335)$, and pharmacists $7 \%(31,872)[4,5]$.

To help the KSA achieve their goals, we projected the needed number of physicians and nurses into 2030. The use of the term "need" in HRH planning, however, remains ambiguous in the literature. In their literature review, Tomblin Murphy and colleagues [6] note that many studies using the term needs-based employ "need", "demand", and "utilization" interchangeably. This circumstance creates a challenge when country stakeholders and academics alike attempt to compare their estimates of HRH to that of other countries (or academics) who use fundamentally different methods and assumptions to arrive at estimates of HRH need [7, 8]. For this reason, we clarify that our needs-based projections use, as the base inputs, estimates of the true prevalence of health conditions (rather than treated prevalence or observed healthcare utilization patterns). Our projections, moreover, are grounded in epidemiology of health conditions and therefore do not consider economic factors such as cost, availability, and willingness to pay.

We developed a new needs-based methodology, rather than using earlier estimates, since previous global ratiobased benchmarks of health worker concentration may not apply to the KSA. Previous estimates of physicians and nurses (per 1000 population) that are needed include 2.28, taken from the World Health Organization (WHO) World Health Report 2006 [9], and 4.5, taken from WHO Sustainable Development Goals 2016 [10]. These benchmarks, however, may not apply to the KSA, given that they were derived from a regression-based exercise using population averages of data on 110 countries, before 2010, and mostly in low- and middle-income contexts with a high burden of infectious diseases. In addition, the 4.5 value assumes a national strategy of universal health coverage in which health care services, even for non-priority health conditions, are subsidized by the federal government. This strategy appears inconsistent with the current restructuring of the Saudi healthcare system and expansion of the private sector [2]. Neither the 4.5 nor the 2.28 estimates, moreover, are based on detailed epidemiologic evidence of the prevalence of health conditions in a particular country. For these reasons, the relevance of these benchmarks to the needsbased estimates of health workers in the KSA remains unknown.

We used an epidemiologic "needs-based" approach to estimate, to 2030, the number of physicians and 
nurses needed to treat the burden of disease in the KSA. This approach has been used previously by governmental and non-governmental organizations [1114] but, in our view, remains underutilized by specific countries. The benefit to the planner of a needs-based approach involves the important goal-setting exercise that answers the following question: what is the expected number of physicians and nurses needed to deliver cost-effective interventions and treatments to adequately address the health needs of the population?

Whereas we focus our forecasting exercise on the KSA, the needs-based methodology can be applied more broadly to other countries intending to efficiently match health workers with the health needs of the changing population. In addition, our needs-based approach innovates this field in 3 ways. First, we forecast the need into the future (i.e., 10-year horizon) using assumptions about the demography of the population. Second, we devise treatment service modules for chronic diseases that impose a large burden of disability and death in and outside of the KSA. Third, we develop a disability-adjusted life year (DALY) multiplier that allows us to scale estimates of health workers to the entire country's burden of disease, rather than to a smaller set of conditions.

\section{Methods}

We proceeded through the following 7 steps to arrive at forecasts of physicians and nurses needed to treat the health conditions of the KSA population to 2030 (see Fig. 1). These steps, adapted from previous literature [14], resulted in 3 different scenarios, each of which considers a range of policy decisions.

(1) We retrieved data on the prevalence of priority health conditions in the KSA, including ischemic heart disease, cerebrovascular disease (stroke), major depressive disorder, diabetes mellitus, chronic obstructive pulmonary disease (COPD), and congenital anomalies, from national health surveys, peer-reviewed literature, and the Global Burden of Disease (GBD) study. (2) We multiplied prevalence estimates by the total population of adults in the KSA to derive the number of cases per condition per year. (3) We assigned treatment coverage goals to each of the 6 priority conditions to estimate the target population that may seek care. (4) We applied service delivery models of cost-effective interventions, based on results of WHO-CHOICE (Choosing Interventions that are Cost-Effective) analyses and cost-effectiveness studies in comparable countries, to estimate outpatient and inpatient visits. (5) We converted the number of visits into full-time equivalent (FTE) heath workers needed to treat priority conditions across settings (i.e., inpatient

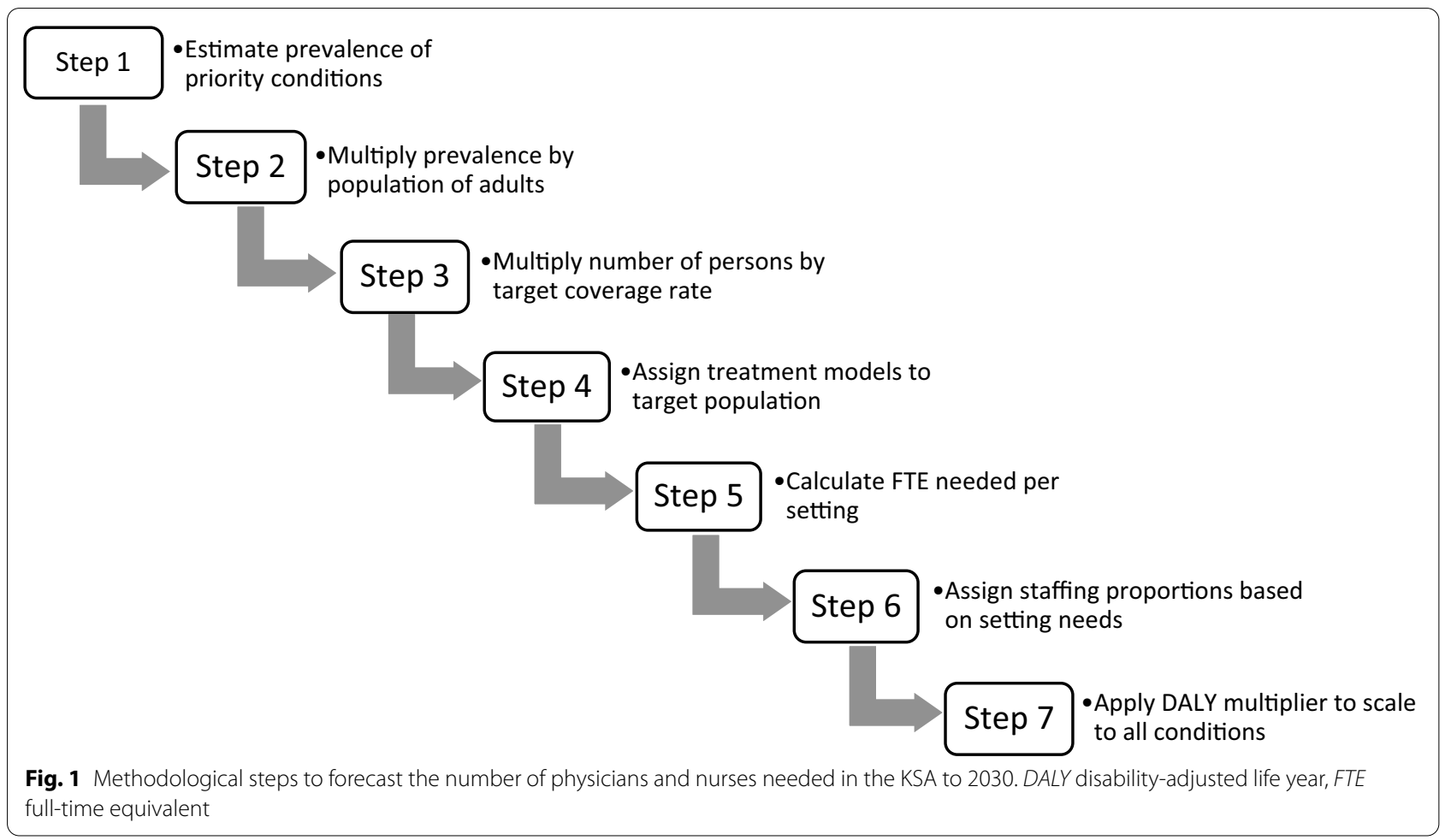


and outpatient) and (6) applied staffing ratios to each treatment setting to obtain quantities of physicians and nurses. (7) We scaled up health workers needed to treat all health conditions in the KSA (i.e., moving beyond the 6 studied priority conditions).

To account for critical uncertainty related to the burden of disease contributed by priority conditions, we applied several DALY multipliers in scale-up models, resulting in 3 different scenarios. We describe each of these steps, and key policy decisions that may influence estimates, in further detail below.

\section{Prevalence of priority conditions}

A needs-based approach uses the prevalence of priority health conditions as its foundation to drive estimates of the size of the health workforce $[11,14-16]$. We therefore used, as the starting point, information on priority health conditions contained in the KSA's Health Sector Transformation Strategy. This strategy document, delivered by the Ministry of Health as part of "Vision 2030" for the KSA, states that, "Particular areas of concern include heart disease, stroke, diabetes mellitus, respiratory disease, mental health, road traffic accidents and congenital diseases..." (p. 9) [17]. Because we expect that preventive measures initiated by 2030 are envisaged to dramatically reduce the number of road traffic accidents, road traffic accidents were excluded for the purposes of this analysis and scenario calculations.

Health conditions within these "areas of concern" were further specified, as the service delivery models required to treat these conditions vary widely. Health conditions were selected according to the following criteria: (a) they impose substantial disability, morbidity, or mortality in the KSA; (b) epidemiologic data and estimates of the condition are available; and (c) the condition is amenable to cost-effective treatment delivered in a primary care or hospital setting. This process resulted in a list of 6 priority health conditions: ischemic heart disease, stroke, major depressive disorder, diabetes mellitus, COPD, and congenital anomalies (including congenital heart defects, neural tube defects, cleft lip and palate).

The best available estimates of prevalence for these 6 priority health conditions, by age group, were used to derive the population that may seek care (Table 1) (Fig. 1, Step 1). Prevalence estimates from national health surveys in the KSA, including the General Authority for Statistics (GASTAT) Household Health Survey [18] and the Saudi Health Interview Survey (SHIS) [19] were prioritized. The GASTAT Household Health Survey, conducted in 2018, is a nationally representative survey targeting all individuals (Saudis and non-Saudis) who reside in the KSA. The survey sample was chosen by identifying 24,012 households that represent the survey population. The SHIS, conducted in 2013, is based on a multistage representative sample of 10,735 adults ages 15 years and older. To ensure estimates are as context relevant as possible, recent data sources were preferred to older ones. For example, for diabetes prevalence, the GASTAT Household Health Survey was preferred to the SHIS as the latter is 5 years older.

Other prevalence estimates including those for COPD and ischemic heart disease rely on Saudi crosssectional, population-based surveys described in peerreviewed literature. For COPD, data were collected between June 2010 and December 2011 using a random digit dialing survey $(n=10,001)$ [20]. A community-based survey, conducted between 1995 and 2000 $(n=17,232)$, was used to estimate the prevalence of ischemic heart disease [21]. In certain cases, where national estimates were not available, the GBD study [22] database was used. Prevalence estimates for the latest year for all ages and both sexes were used.

\section{Total cases}

Prevalence estimates for priority health conditions were then multiplied by the United Nations population projections for years 2020-2030 [23] to derive possible cases per condition per year (Fig. 1, Step 2). This calculation assumes no change in prevalence of these conditions to 2030.

\section{Treatment coverage targets}

Information on plausible treatment coverage rates was then applied to each of the priority health conditions (Fig. 1, Step 3). Target coverage rates were determined on the basis of the severity of the health condition, the ability of a clinician to detect cases, and the probability that cases will seek care. Based on these factors, and consistent with the approach used in the literature in devising these targets [24-26], the following treatment coverage rates were established (and applied across all years): $80 \%$ for ischemic heart disease, stroke, COPD, diabetes mellitus, and congenital anomalies; and 33\% for major depressive disorder. A relatively high treatment coverage target was assigned to cardiovascular diseases, COPD, diabetes mellitus, and congenital anomalies due to the large burden associated with these conditions in the KSA and, for acute events, the severity of symptoms and ease of detection. Conversely, a relatively low target coverage rate was assigned to major depressive disorder given that patients may not report symptoms and clinicians in a primary care setting may fail to detect cases [27]. We also assessed the potential impact of a health education campaign that 
Table 1 Population-based estimates of prevalence for priority health conditions and their sources

\begin{tabular}{lll}
\hline Priority health condition & Prevalence (\%) & Source \\
\hline Ischemic heart disease & 5.50 & Al-Nozha et al. [21] \\
Cerebrovascular disease & 0.65 & GBD study 2017 [22] \\
Major depressive disorder & 2.78 & GBD study 2017 [22] \\
Diabetes mellitus & 8.50 & GASTAT 2018 [18] \\
COPD & 2.40 & Wali et al. [20] \\
Congenital anomalies & 1.24 & GBD study 2017 [22] \\
\hline
\end{tabular}

COPD chronic obstructive pulmonary disease, GASTAT General Authority for Statistics, GBD Global Burden of Disease

would increase treatment coverage levels (see "Scenarios and policy decisions" below).

\section{Service delivery models}

Next, for each condition, we applied service delivery models of cost-effective interventions to estimate the number of outpatient and inpatient visits per year (Table 2) (Fig. 1, Step 4). These models were based on results of WHO-CHOICE [28] regional analyses and cost-effectiveness studies in upper-middle- and highincome countries, which best reflect the economic circumstance of the KSA [14, 24, 26]. Treatment models were determined based on (a) the percentage of cases needing care in each service setting (i.e., inpatient and outpatient), (b) the average annual number of visits per person, and (c) whether or not visits require a bed (see Additional file 1: Table S2). The WHO-CHOICE [28] cost-effective modules assume that most cases receive treatment at a primary care level, and that patients with more severe, complex, or acute conditions receive treatment at a hospital level.

\section{Health workforce staffing}

Using these outpatient and inpatient visit estimates, treatments were converted into the FTE number of physicians and nurses needed to treat priority health conditions
(Fig. 1, Step 5). Workforce requirements for outpatient services were calculated using WHO estimates of workforce capacity $[14,28]$. Models assumed that physicians and nurses work, on average, 225 days per year and have 11 consultations per day. The total number of expected outpatient visits was divided by $2475(225 \times 11)$ to obtain an estimate of the number of FTE physicians and nurses needed for outpatient care. To derive the number of FTE physicians and nurses required to meet inpatient needs, bed-days were estimated assuming that hospitals operate at $85 \%$ capacity [14]. This correction factor (1.15) was applied to obtain the target number of inpatient beddays. Models assumed no change in FTE inputs across years.

\section{Staffing ratios}

Staffing ratios were then applied to each treatment setting. Models assumed that physicians and nurses perform $1.7 \%$ and $98.3 \%$ of tasks in outpatient settings, and $10 \%$ and $90 \%$ of tasks in inpatient settings, respectively (Fig. 1, Step 6) [14, 24, 28]. We also assessed how task-shifting (i.e., where tasks are shifted from physicians to nurses) would affect needs-based health worker estimates (see "Scenarios and policy decisions" below).

\section{Scale-up models}

Health workers were then scaled up to treat all health conditions by applying a DALY multiplier to the subset of priority health conditions studied (Fig. 1, Step 7). Scaleup models assumed that, on average, the health workforce required to treat a health condition is proportional to its contribution to the total burden of disease, as measured by DALYs, in the KSA.

This assumption was derived from data on the use of health services, retrieved from the FutureDocs Forecasting Tool [29], and the GBD study [22] for select health conditions in the US and KSA. The FutureDocs Forecasting Tool estimates the capacity of the health workforce to meet the use of health services in the US from 2011

Table 2 Needs-based estimates of outpatient visits and inpatient bed-days for priority health conditions in the KSA, 2030

\begin{tabular}{lccc}
\hline Health condition & Target population & Total annual outpatient visits & $\begin{array}{c}\text { Total annual } \\
\text { inpatient bed- } \\
\text { days }\end{array}$ \\
\hline Cardiovascular diseases & $1,525,397$ & $3,050,794$ & $3,050,794$ \\
Major depressive disorder & 284,431 & $1,498,950$ & 207,634 \\
Diabetes & $2,108,272$ & $4,216,544$ & 843,309 \\
COPD & 595,277 & $1,506,050$ & 357,166 \\
Congenital anomalies & 25,544 & 6,386 & 109,839 \\
Total & $4,538,921$ & $10,278,724$ & $4,568,742$ \\
\hline
\end{tabular}

COPD chronic obstructive pulmonary disease 
to 2030 [29]. We compared the proportion of inpatient and outpatient visits, as a percentage of all visits [29], to the proportion of the burden of disease, as a percentage of the total burden of disease [22], for several priority conditions. For example, respiratory diseases (e.g., COPD) cause (a) 95,683,118 inpatient and outpatient visits (6.83\% of total visits), and (b) 6,465,807 DALYs (6.43\% of total DALYs); and mental disorders (e.g., major depression) cause (a) 75,235,774 inpatient and outpatient visits (5.37\% of total visits) and (b) 5,371,622 DALYs (5.34\% of total DALYs) in the US per year. Additional examples demonstrating the proportionality assumption underlying the use of a DALY multiplier to scale up health workers appear in the Additional file 1: Table S3.

Using information available in peer-reviewed literature [30] and results of the GBD study [22], the 6 studied priority health conditions in the KSA were estimated to account for $20 \%$ of the total burden of disease in the KSA. Based on the proportionality assumption, the number of FTE staff needed to treat priority conditions was multiplied by a factor of 5 to obtain the total number of FTE health workers required to treat all health conditions.

\section{Alternative scenarios and policy decisions}

We projected, from 2020 to 2030, needs-based health worker estimates under various assumptions about scaling, resulting in 3 scenarios. Within each scenario, we further assessed the potential role of policy decisions related to task-shifting and treatment coverage rates on the estimated number of physicians and nurses needed to treat the epidemiologic needs of the population. These scenarios and policy decisions are intended to provide planners and policymakers with a range of health workforce planning options-of which a subset may be feasible given political and economic constraints.

\section{Scenarios}

The DALY multiplier of 5 (Scenario A) assumes that the burden of disease contributed by the 6 priority conditions accounts for $20 \%$ of the total burden of disease in the KSA [22, 30]. We applied 2 additional DALY multipliers (4 and 6) to account for critical uncertainty related to this assumption. This process resulted in 3 different scenarios, where multiplication by a factor of 5 produces a median, or baseline, estimate of required health workers (Scenario A), and 4 (Scenario B) and 6 (Scenario C) provide a plausible range of health workers based on underor overestimates of the burden of disease contributed by priority conditions.

\section{Policy decisions}

Staffing ratios Next, alternative staffing ratios, which assume that a percentage of tasks $(30 \%$ or $50 \%)$ can be "shifted" from physicians to nurses, were applied. Staffing ratios based on task-shifting decisions were adjusted relative to the baseline staffing ratios described above. For instance, if $30 \%$ of tasks were shifted from physicians to nurses, then physicians would perform approximately $1.2 \%$ of tasks in outpatient settings and $7 \%$ of tasks in inpatient settings.

Treatment coverage rates Lastly, a speculative policy option was considered, in which a health education campaign would increase absolute treatment coverage rates for all health conditions by $10 \%$. The assumption of this policy is that persons with these conditions would be more likely to seek care. The resulting target coverage rates include $90 \%$ for ischemic heart disease, stroke, COPD, diabetes mellitus, and congenital anomalies, and $43 \%$ for major depressive disorder.

Modeling all possible combinations of assumptions and corresponding inputs resulted in 3 scenarios (3 DALY multipliers), and 6 policy decisions ( 3 staffing ratios $\times 2$ levels of target coverage) per scenario, totaling 18 workforce estimates (see schematic diagram in Fig. 2).

\section{Results}

\section{Baseline model}

Our baseline needs-based estimates (Model 1) are based on Scenario A (DALY multiplier of 5) and do not account for policy decisions that may increase task-shifting and/ or treatment coverage levels. Specifically, the baseline model assumes that (a) priority health conditions account for $20 \%$ of the total burden of disease in the KSA, (b) no task-shifting occurs, and (c) $80 \%$ of expected cases receive treatment for cardiovascular diseases, diabetes, COPD, and congenital anomalies, and 33\% for major depressive disorder.

Under these assumptions, approximately 64,000 FTE nurses and physicians are needed in 2020 to meet epidemiological needs. Using the needs-based approach, it is estimated that, of these FTE workers, 4935 physicians and 59,054 nurses are required. These values equate to 2.01 health workers (physicians and nurses) per 1000 population. When projected to 2030, this FTE estimate rises to approximately 75,000 workers (5788 physicians and 69,399 nurses). This workforce equates to 2.05 physicians and nurses per 1000 population in 2030. Figure 3 provides an overview of needs for each year, between 2020 and 2030, based on Model 1.

\section{Alternative scenarios and policy decisions}

Models that take into account different scenarios and policy decisions reveal that the baseline needs for FTE physicians and nurses could be close to 75,000 , with a 


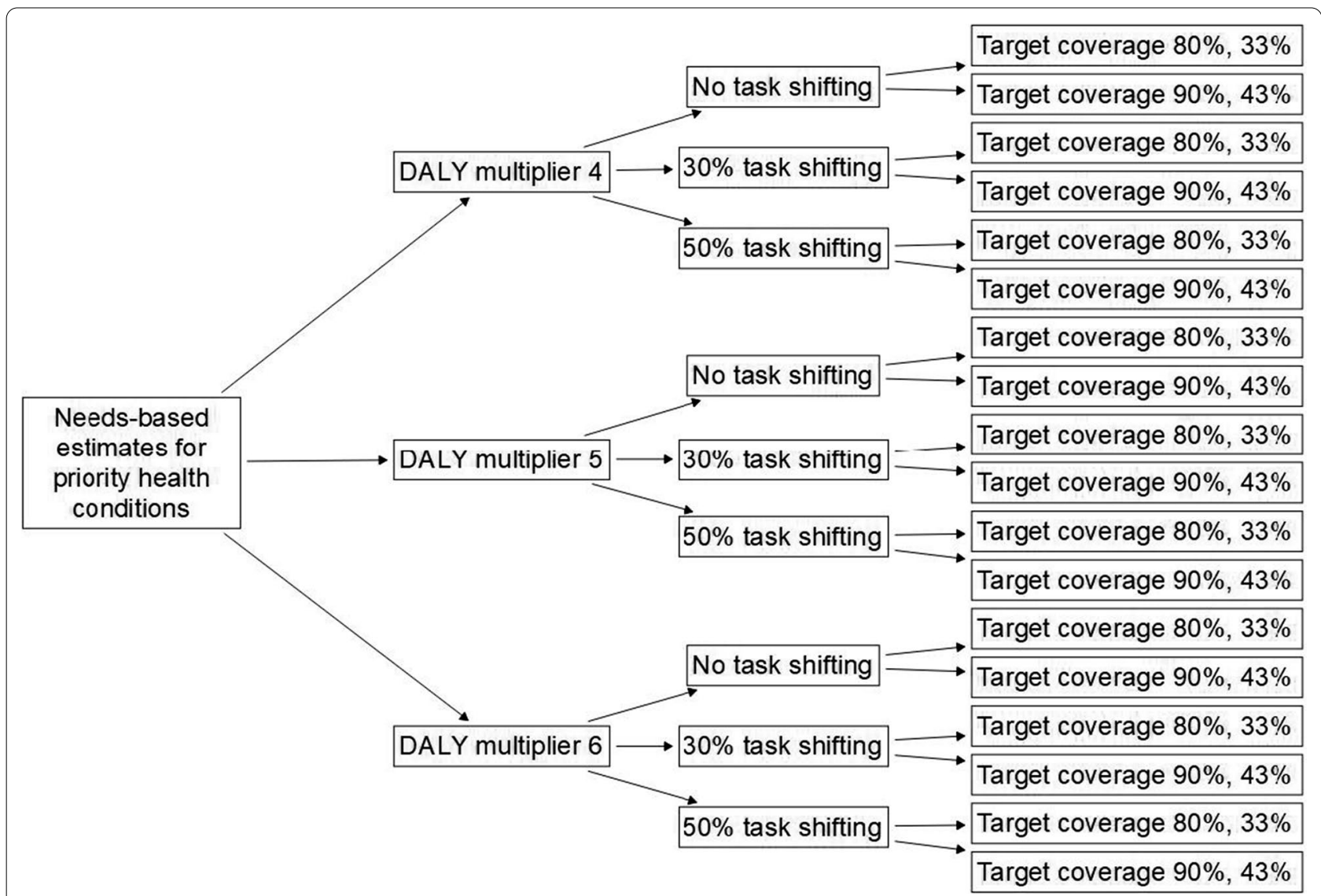

Fig. 2 Schematic diagram of 18 needs-based estimates under various assumptions of scaling, task-shifting, and target coverage level. DALY disability-adjusted life years

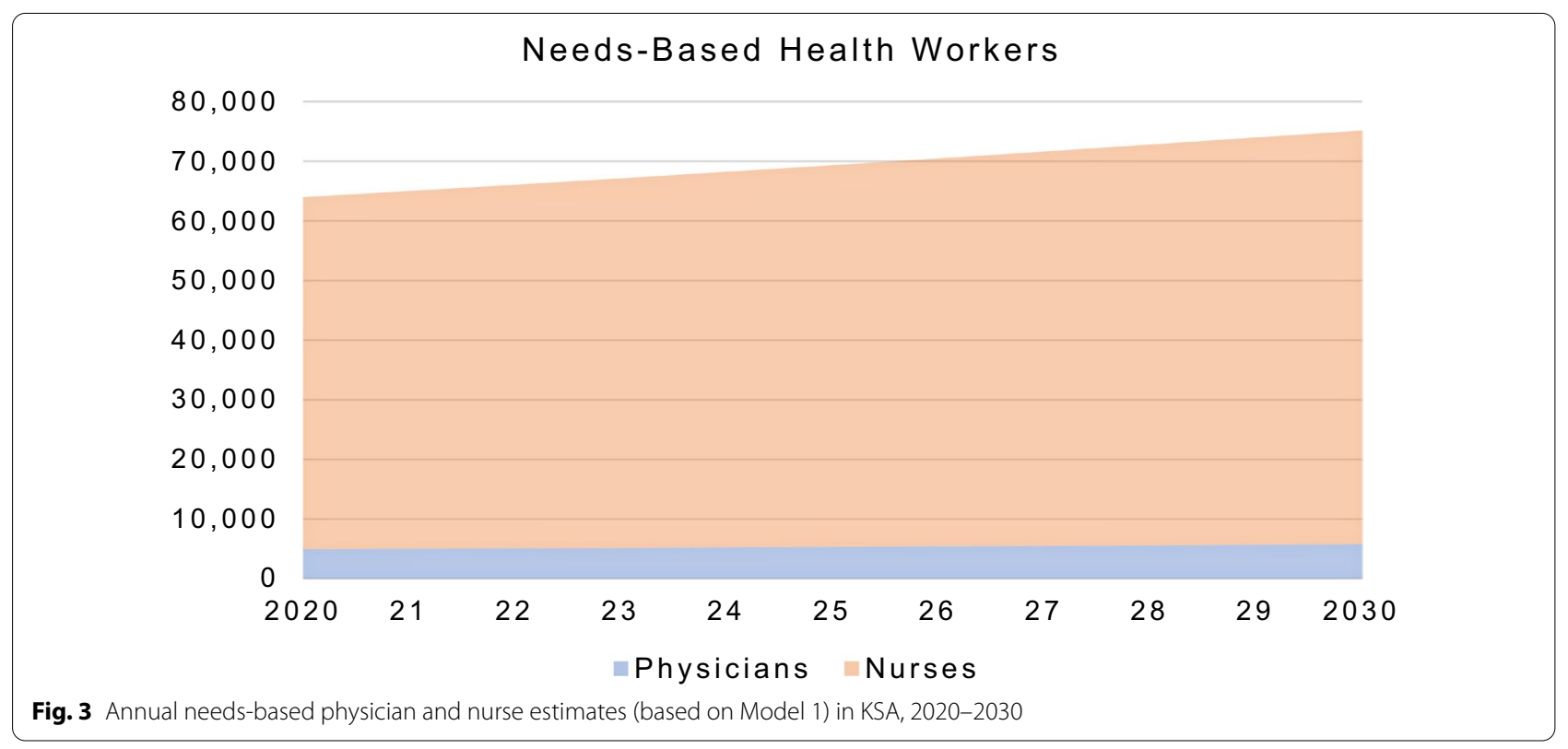


Table 3 Needs-based estimates of the number of FTE physicians and nurses in 2030 according to model assumptions

\begin{tabular}{|c|c|c|c|c|c|c|c|c|}
\hline \multirow{2}{*}{\multicolumn{2}{|c|}{$\frac{\text { Scenario }}{\text { DALY multiplier }}$}} & \multirow[b]{2}{*}{ Model } & \multicolumn{2}{|c|}{ Policy decisions } & \multicolumn{4}{|c|}{ Needs-based health workers } \\
\hline & & & Task-shifting & Target coverage & Physicians & Nurses & Total & $\begin{array}{l}\text { Per } 1000 \\
\text { pop }\end{array}$ \\
\hline \multirow{6}{*}{$\begin{array}{l}\text { A } \\
\text { Median }\end{array}$} & \multirow[t]{6}{*}{5} & $1^{*}$ & - & - & 5788 & 69,399 & 75,187 & 2.05 \\
\hline & & 2 & - & $+10 \%$ & 6989 & 86,412 & 93,401 & 2.54 \\
\hline & & 3 & $30 \%$ & - & 4052 & 71,135 & 75,187 & 2.05 \\
\hline & & 4 & $30 \%$ & $+10 \%$ & 4892 & 88,508 & 93,401 & 2.54 \\
\hline & & 5 & $50 \%$ & - & 2894 & 72,293 & 75,187 & 2.05 \\
\hline & & 6 & $50 \%$ & $+10 \%$ & 3494 & 89,906 & 93,401 & 2.54 \\
\hline \multirow{6}{*}{$\begin{array}{l}\text { B } \\
\text { Lower limit }\end{array}$} & \multirow[t]{6}{*}{4} & 7 & - & - & 4631 & 55,519 & 60,150 & 1.64 \\
\hline & & 8 & - & $+10 \%$ & 5591 & 69,129 & 74,720 & 2.03 \\
\hline & & 9 & $30 \%$ & - & 3241 & 56,908 & 60,150 & 1.64 \\
\hline & & 10 & $30 \%$ & $+10 \%$ & 3914 & 70,807 & 74,720 & 2.03 \\
\hline & & 11 & $50 \%$ & - & 2315 & 57,835 & 60,150 & 1.64 \\
\hline & & 12 & $50 \%$ & $+10 \%$ & 2796 & 71,925 & 74,720 & 2.03 \\
\hline \multirow{6}{*}{$\begin{array}{l}\text { C } \\
\text { Upper limit }\end{array}$} & \multirow[t]{6}{*}{6} & 13 & - & - & 6946 & 83,279 & 90,225 & 2.46 \\
\hline & & 14 & - & $+10 \%$ & 8387 & 103,694 & 112,081 & 3.05 \\
\hline & & 15 & $30 \%$ & - & 4862 & 85,363 & 90,225 & 2.46 \\
\hline & & 16 & $30 \%$ & $+10 \%$ & 5871 & 106,210 & 112,081 & 3.05 \\
\hline & & 17 & $50 \%$ & - & 3473 & 86,752 & 90,225 & 2.46 \\
\hline & & 18 & $50 \%$ & $+10 \%$ & 4193 & 107,887 & 112,081 & 3.05 \\
\hline
\end{tabular}

$D A L Y$ disability-adjusted life years, pop population

*Results of primary baseline model

plausible range of between 60,000 and 112,000, in 2030 (see Table 3). Changes in the DALY multiplier reflect changes to the percentage of the total burden of disease accounted for by priority health conditions. Figure 4 shows the range of estimates produced under different assumptions about scaling, where Scenarios B and C produce upper- and lower-limits of estimated needs-based health workers, respectively.

Some models within each scenario consider the role of a policy decision that would increase task-shifting-that is, enhancements in certifications or in training among nurses may increase task-shifting to between 30 and 50\%, reducing the ratio of physicians to nurses. For example, under Scenario A, enacting a 30\% task-shifting policy (Model 3) produces needs-based estimates of 4052 physicians and 71,135 nurses. Some models also account for the potential impact of an enhanced public health campaign. Model 2, which assumes a 10\% increase in treatment coverage under Scenario A, for example, produces needs-based estimates of 6989 physicians and 86,412 nurses.

\section{Discussion}

Regression-based estimates of health worker densities that are required to treat the epidemiologic needs of a population are limited in that they typically ignore the specific country's demographic age structure, prevalence of health conditions, and workforce planning goals. Here we detail a needs-based methodology to calculate, and forecast, the number of physicians and nurses needed to treat a country's health conditions. We focused on the KSA because this country aims to achieve transformational goals for its health workforce by 2030 . These methods, however, can be widely applied to other countries interested in estimating workforce needs based on the prevalence of health conditions in their population.

The needs-based modeling in the KSA shows that the needs for FTE physicians and nurses in 2030 range between 60,000 and 112,000 depending on the scenario and policies enacted; however, we expect the actual need is closer to 75,000 health workers. This range translates into physician and nurse densities from 1.64 to 3.05 per 1000 population. These densities fall within the general range of earlier ratio-based benchmarks reported by WHO in the World Health Report 2006 (i.e., 2.28) and the Universal Health Coverage Report of 2016 (i.e., 4.5) $[9,10]$. Importantly, the current modeling exercise provides a level of granularity to the KSA and uses detailed epidemiologic estimates for one country. For this reason, the current needs-based exercise for the KSA represents 


\section{a Needs-Based Physicians}

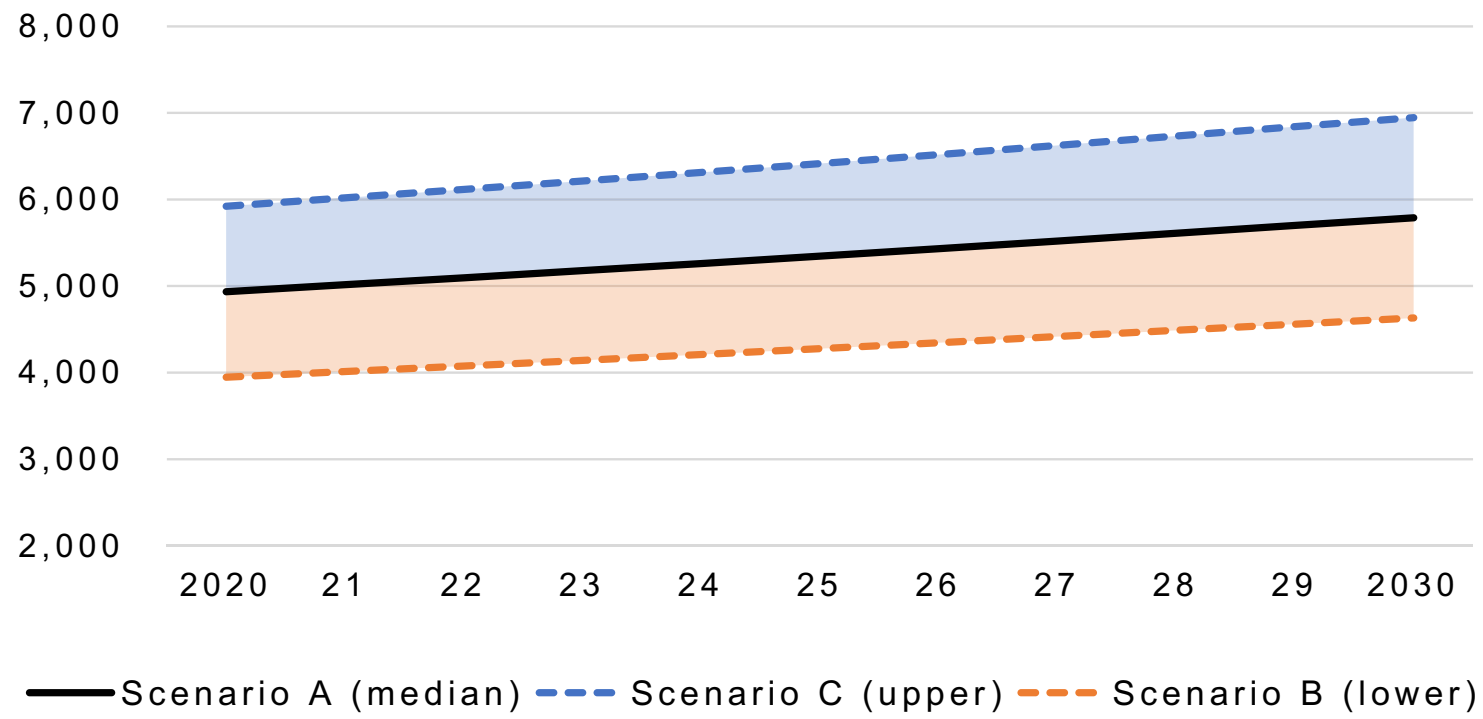

\section{b Needs-Based Nurses}

90,000

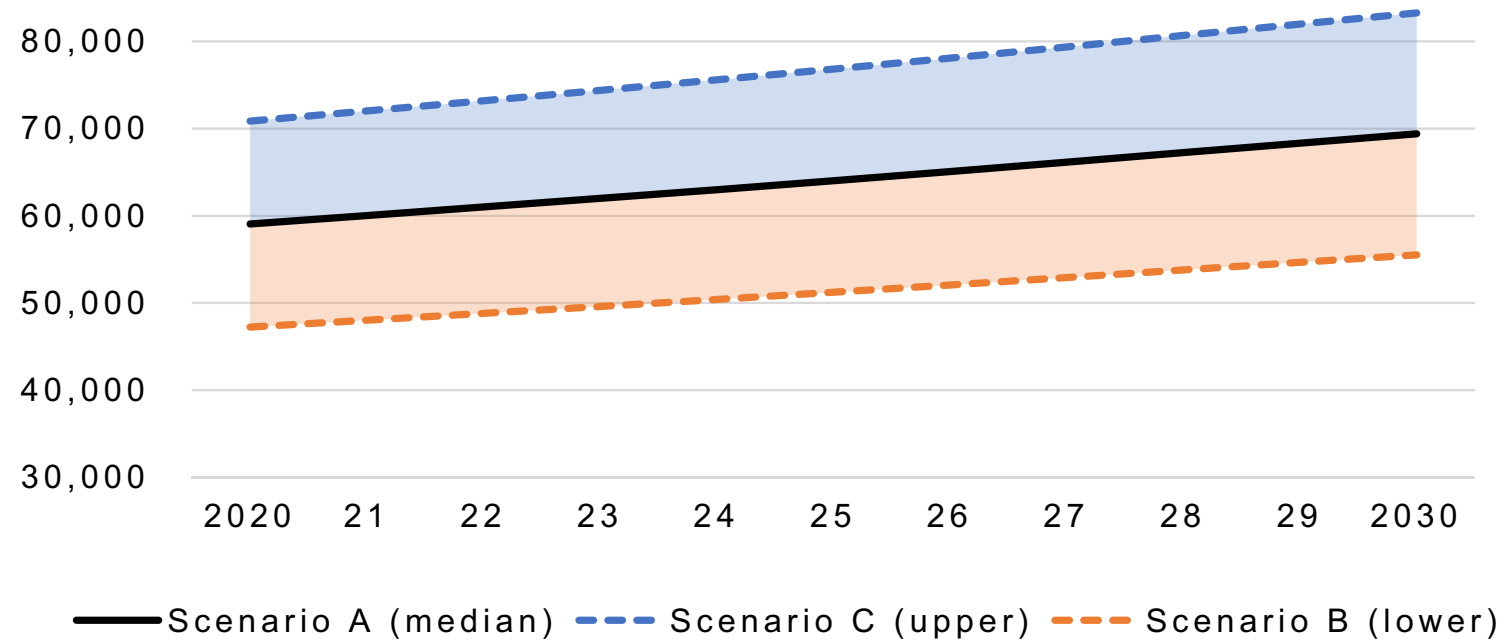

Fig. 4 Annual needs-based $\mathbf{a}$ physician and $\mathbf{b}$ nurse estimates and scenario-based ranges* in the KSA, 2020-2030. *Scenario estimates do not include policy decisions; Scenario A = Model 1, Scenario B = Model 7, Scenario C= Model 13

a substantial improvement relative to the use of population-level regression averages that rely on low- and middle-income country contexts and assume a social goal of universal health coverage for all.

The estimated number of FTE health workers needed is below the total stock of physicians and nurses in the KSA, but above the stock of Saudi national physicians and registered nurses. According to recent data $[4,5]$, when counting both Saudi and non-Saudi nationals, as well as registered/bachelor and enrolled/diploma nurses, there are approximately 300,000 physicians and nurses in the KSA, which equates to about 9 health care workers per 1000 population. This estimated health workforce exceeds the needs-based estimates provided above (though the needs-based approach does not account for economic factors that may also drive the supply of 
workers). At the same time, the needs-based estimate does not exceed current stock if accounting only for Saudi nationals and separating registered nurses from diploma nurses (i.e., those who do not have a college degree in nursing). Only about 100,000 of the physicians and nurses are Saudi, which equates to about 3 physicians and nurses per 1000 population. Without diploma nurses, the density of Saudi physicians and nurses is only 1.4 per 1000 population.

Whereas the KSA may not face an overall shortage of physicians and nurses in 2030, the literature suggests that a shortfall of general practitioners remains possible $[4$, 5]. Approximately $22 \%$ of the KSA's physician workforce classify as generalists (defined as general practitioners or family medicine physicians working in primary care) and $74 \%$ as specialists. By contrast, in OECD countries, on average, $30 \%$ and $63 \%$ of physicians work as generalists and specialists, respectively $[4,5]$.

Additionally, the KSA may face a mismatch among specialists, where some specialties appear overrepresented relative to demand. For example, the KSA has above average levels of surgical specialists, obstetricians/gynecologists (OBGYNs), and pediatricians, but below average levels of psychiatrists and endocrinologists. Post-graduate training, moreover, is skewed toward specialties with current overcapacity. To address the potential shortage of primary care doctors and achieve its strategic objectives, the KSA may need to increase its share of generalists (e.g., from $22 \%$ to nearer the OECD average of $30 \%$ ) and encourage growth in underrepresented specialties $[4,5]$.

Limitations of needs-based estimates include that, while they are useful for workforce planning, they rely on several assumptions. A key assumption is that the "input" data used (e.g., on prevalence of conditions, treatment coverage, worker productivity) are accurate. Additionally, models are initially based on prevalence estimates of 6 priority health conditions that account for approximately $20 \%$ of the disease burden and workforce effort in the KSA $[22,30]$. This approach may yield different results than one which attempts to enumerate the disease burden for each and every condition that affects the Saudi population, or an approach that uses as its basis a set of conditions that require a greater share of workforce effort. Moreover, we applied a range of DALY multipliers to account for inherent uncertainty in scale-up models. However, future work may benefit from assessing the validity of alternative DALY multipliers based on the capacity of the health workforce to meet the health needs of the population (e.g., a less efficient workforce may require more physicians and nurses to treat the same disease burden).

We also note a scarcity of evidence on cost-effective staffing ratios, particularly in allied health professions
[31]. For this reason, and to simulate the influence of policies that encourage task-shifting, we applied several staffing ratios. However, the most cost-efficient and effective ratio of physicians to nurses likely varies by healthcare setting, country, and condition. Future research that examines workforce ratios, particularly in the growing areas of mental health services and diabetes care, would be useful for planning health workforce requirements into the future.

In addition, we cannot compare treatment coverage goals set for each priority condition to current levels of coverage in the KSA. Although information on condition-specific treatment coverage is limited, the KSA does provide recent estimates of the total percentage of the population covered by the healthcare system, by age group and nationality (Saudi/non-Saudi) [18]. According to these data, in $201838.6 \%$ of the total population of the KSA (i.e., across age group and nationality) had health insurance coverage [18]. Our condition-specific treatment coverage goals exceed overall healthcare coverage in the KSA, but treatment coverage likely varies substantially by condition. Planners would benefit from having information on treatment coverage by health condition, rather than in the aggregate, so that policymakers could set reasonable goals for gradually improving treatment coverage for priority conditions.

All of the scenarios modeled in the KSA assume no worsening in the burden of chronic disease by 2030, other than due to population ageing, which may be optimistic. Several projections about the burden of chronic disease in the KSA into 2030 and beyond portend an increase in diabetes, stroke, and heart disease, among other conditions [32, 33]. These increases may arise in part from the ageing of the population and continued increases in obesity-related chronic diseases. To the extent that these trends continue, the overall prevalence of heart disease, stroke, and diabetes could increase over time, and our workforce needs-based estimates might therefore be underestimated, regardless of the other assumptions made. Careful attention and monitoring are warranted to understand these conditions into the future. If these conditions continue to increase in prevalence as expected, then policy efforts that focus specifically on cardiovascular diseases and diabetes may be necessary.

Additionally, when we developed our needs-based methodology, the coronavirus disease (COVID-19) did not contribute substantially to the current or anticipated disease burden in the KSA. Accounting for the COVID19 pandemic may change the number of physicians and nurses needed to treat the unique epidemiologic needs of the population in 2020-2021. However, communicable diseases, like traffic accidents (as discussed above in "Background"), appear more amenable to reduction 
through population-level prevention strategies and public health efforts than through health care treatments. We expect that, with improved population-level prevention and widespread vaccination, the pandemic will not affect the 2030 forecast.

Planners should also be reminded that estimates do not account for patient preferences. In the KSA context, patients may prefer a particular gender, Saudi national vs. foreign health provider, nurse vs. physician, or resident vs. consultant. In this exercise, we assumed that health care worker productivity can be applied equally to all patients regardless of preferences. This assumption may be politically and socially untenable and/or undesirable for the Saudi population from a "demand-side" perspective.

The important question of ideal worker "mix" remains an issue in the KSA and elsewhere. There remain unresolved questions about the benefit/cost calculations of employing and training physicians vs. nurses, the optimal level of task-shifting, the quality of training programs for various worker specialties, and (in some countries) goals to reduce the reliance on foreign workers. All of these issues represent important policy and economic considerations. Our needs-based estimates, therefore, should be placed within the larger context of the mix of public and private investments, as well as patient preferences, regarding what gender, worker type, and skill mix would be ideal for a particular country.

Wealthy neighboring countries with similar demographics to the KSA may benefit from this analysis. The Gulf Cooperation Council (GCC) countries share common challenges associated with health care workers' availability and distribution [34-36]. Furthermore, evidence shows very similar patterns of non-communicable diseases and associated risk factors in the GCC countries [37-40]. This approach may therefore be utilized as a viable tool for anticipating the right number of physicians and nurses needed into the future in any other member country of the GCC.

\section{Conclusion}

Needs-based forecasts provide a visioning exercise in that they attempt to answer the question: how many workers would it take to achieve a desired level of healthcare coverage for all members of society? Our baseline model shows that approximately 75,000 workers (5788 physicians and 69,399 nurses) -2.05 workers per 1000 population-will be needed to meet the epidemiologic needs of the Saudi population in 2030. Based on this projection, the KSA will not face a needs-based health worker shortage in 2030. However, alternative estimates based on scenarios assuming different levels of population health, and policy decisions that take into account changes in task-shifting and enhanced public health campaigns, suggest that the actual needs for FTE physicians and nurses could range from 60,000 (1.64 per 1000 population) to 112,000 (3.05 per 1000 population).

Estimates of needs-based workforce densities depend strongly on how need is defined [6, 41]. Previous HRH literature, for instance, has used need to describe not only the health workforce required to treat the population burden of disease, but also patient demand, willingness to pay, and health care supply. Our approach and our definition of "needs-based" applies a population lens that focuses on the overall disease burden and may promote agendas. However, this approach contrasts economic, or "willingness to pay," approaches which consider the economic aspects of price, supply, and demand [6, 7]. Our approach also assumes equitable and efficient allocation of health services to all, which in some countries may not be realistic.

To this end, our needs-based approach should be viewed as epidemiological, rather than economic. Where country-level data are available, we encourage the use of needs-based forecasts as a complement to labor market demand-based forecasts. Furthermore, integration of preventive and public health efforts as part of the Vision 2030 Strategy, which focuses on the social and economic determinants of health, may crucially affect workforce need. Taken together, these multi-sector strategies will assist with setting appropriate workforce goals and planning accordingly for 2030.

\section{Abbreviations}

COPD: Chronic obstructive pulmonary disease; DALYs: Disability-adjusted life years; FTE: Full-time equivalent; GASTAT: General Authority for Statistics; GBD: Global Burden of Disease; GCC: Gulf Cooperation Council; GHDx: Global Health Data Exchange; KSA: Kingdom of Saudi Arabia; SHIS: Saudi Health Interview Survey; WHO: World Health Organization.

\section{Supplementary Information}

The online version contains supplementary material available at https://doi. org/10.1186/s12960-021-00597-w.

Additional file 1: Table S1. All population-based estimates of prevalence for priority health conditions (color-coded by data source). Table S2. Service delivery model exemplars for priority conditions. Table S3. Use of health services and burden of disease for selected health conditions in the United States.

\section{Acknowledgements}

The paper was produced by the Saudi Health Council, with technical support from individuals from the University of California, Irvine, and the World Bank.

The authors are grateful for the guidance and advice on the drafts provided by Ziad M Nakshabandi, CEO of National Center for Health Workforce Planning at the Saudi Commission for Health Specialties. The authors are also grateful for the support provided by Sameh El-Saharty, World Bank Human Development Program Lead, GCC countries, and Rekha Menon, World Bank Practice Manager, Health Nutrition and Population, Middle East and North Africa region. 


\begin{abstract}
Authors' contributions
MA, TA, CHH, JXL, TKL and TAB conceived the idea; MA, TA, HA, NT, NA, and $\mathrm{MH}$ procured the relevant datasets; SG and TAB led the analysis; $S G$ and TAB drafted the manuscript. All authors reviewed the manuscript for intellectual content, contributed to writing sections of the manuscript. All authors read and approved the final manuscript.
\end{abstract}

\section{Funding}

Financing was provided by the Saudi Health Council and under the Health Nutrition and Population Reimbursable Advisory Services Program (P172148) between the World Bank and the Ministry of Finance in Saudi Arabia.

\section{Availability of data and materials}

Not applicable.

\section{Declarations}

Ethics approval and consent to participate

Not applicable.

\section{Consent for publication}

Not applicable.

\section{Competing interests}

The authors declare that they have no competing interests. The findings, interpretations, and conclusions expressed in this work are those of the authors, and do not necessarily reflect the views of The Saudi Council and the World Bank, their Board of Directors, or the governments they represent.

\section{Author details}

${ }^{1}$ School of Social Ecology, University of California Irvine, Irvine, CA, USA. ${ }^{2}$ Program in Public Health, University of California Irvine, Irvine, CA, USA. ${ }^{3}$ Department of Clinical Pharmacy, Medication Outcomes Center, University of California San Francisco, San Francisco, CA, USA. ${ }^{4}$ Department of Social and Behavioral Sciences, Institute for Health and Aging, University of California San Francisco, San Francisco, CA, USA. ${ }^{5}$ Saudi Health Council, Riyadh, Saudi Arabia. ${ }^{6}$ Lancaster University, Lancashire, UK. ${ }^{7}$ Ministry of National GuardHealth Affairs, Riyadh, Saudi Arabia. ${ }^{8}$ World Bank, Washington, DC, USA.

Received: 17 December 2020 Accepted: 31 March 2021 Published online: 26 April 2021

\section{References}

1. National transformation program: delivery plan 2018-2020. In: Saudi Vision 2030. 2018. https://vision2030.gov.sa/sites/default/files/attac hments/NTP\%20English\%20Public\%20Document_2810.pdf. Accessed 1 Nov 2019.

2. Walston S, Al-Harbi Y, Al-Omar B. The changing face of healthcare in Saudi Arabia. Ann Saudi Med. 2008;28:243-50.

3. Key Health Indicators, 2019 Health Indicators. https://www.moh.gov.sa/ en/Ministry/Statistics/Indicator/Pages/Indicator-1440.aspx.

4. Nurses. In: Health resources. Organisation for Economic Cooperation and Development (OECD). https://doi.org/10.1787/283e64de-en. Accessed 8 May 2020.

5. Doctors. In: Health resources. Organisation for Economic Cooperation and Development (OECD). https://doi.org/10.1787/4355e1ec-en. Accessed 8 May 2020

6. Tomblin Murphy G, Birch S, MacKenzie A, Bradish S, Elliott RA. A synthesis of recent analyses of human resources for health requirements and labour market dynamics in high-income OECD countries. Hum Resour Health. 2016;14(1):59.

7. Murphy GT, MacKenzie A, Guy-Walker J, Walker C. Needs-based human resources for health planning in Jamaica: using simulation modelling to inform policy options for pharmacists in the public sector. Hum Resour Health. 2014;12(1):1-11.

8. Murphy GT, Gilbert JH, Rigby J. Integrating interprofessional education with needs-based health workforce planning to strengthen health systems. J Interprof Care. 2019;33(4):343-6.
9. World Health Organization. The world health report 2006: working together for health. 2006. https://www.who.int/whr/2006/en/. Accessed 1 May 2020.

10. World Health Organization. Health workforce requirements for universal health coverage and the sustainable development goals. 2016. https:// www.who.int/hrh/resources/health-observer17/en/. Accessed 7 May 2020.

11. Liu JX, Goryakin Y, Maeda A, Bruckner T, Scheffler R. Global health workforce labor market projections for 2030. The World Bank; 2016.

12. Scheffler RM, Liu JX, Kinfu Y, Dal Poz MR. Forecasting the global shortage of physicians: an economic-and needs-based approach. Bull World Health Organ. 2008;86:516-23.

13. Asamani JA, Christmals CD, Reitsma GM. The needs-based health workforce planning method: a systematic scoping review of analytical applications. Health Policy Plann. 2021. https://doi.org/10.1093/heapol/ czab022.

14. Bruckner TA, Scheffler RM, Shen G, Yoon J, Chisholm D, Morris J, et al. The mental health workforce gap in low- and middle-income countries: a needs-based approach. Bull World Health Organ. 2011;89:184-94.

15. Scheffler RM, Mahoney CB, Fulton BD, Dal Poz MR, Preker AS. Estimates of health care professional shortages in sub-Saharan Africa by 2015. Health Aff. 2009;28:w849-62

16. McPake B, Scott A, Edoka I. Analyzing markets for health workers: insights from labor and health economics. Washington: World Bank Publications; 2014.

17. The need for transformation. In: Health sector transformation strategy. Ministry of Health (Kingdom of Saudi Arabia). 2017. https://www.moh. gov.sa/en/Ministry/vro/Documents/Healthcare-Transformation-Strategy. pdf. Accessed 1 Nov 2019.

18. GASTAT. 2018. Household Health Survey. https://www.stats.gov.sa/en/ 965.

19. IHME. 2013. Saudi Health Interview Survey. http://www.healthdata.org/ $\mathrm{ksa} /$ projects/saudi-health-interview-survey.

20. Wali S, Idrees M, Alamoudi O, Aboulfarag A, Salem A, Aljohaney A, et al. Prevalence of chronic obstructive pulmonary disease in Saudi Arabia. Saudi Med J. 2014:35:684-90.

21. Al-Nozha MM, Arafah M, Al-Mazrou YY, Al-Maatouq MA, Khan NB, Khalil MZ, et al. Coronary artery disease in Saudi Arabia. Saudi Med J. 2004;25(9):1165-71.

22. Global Burden of Disease Study 2017. GDB 2017. Seattle, United States: Institute for Health Metrics and Evaluation (IHME). 2018.

23. United Nations, Department of Economic and Social Affairs, Population Division; 2019; World Population Prospects.

24. Chisholm D, Lund C, Saxena S. Cost of scaling up mental healthcare in low-and middle-income countries. Br J Psychiatry. 2007;191:528-35.

25. Ortegón M, Lim S, Chisholm D, Mendis S. Cost effectiveness of strategies to combat cardiovascular disease, diabetes, and tobacco use in subSaharan Africa and South East Asia: mathematical modelling study. BMJ. 2012;344:e607.

26. Salomon JA, Carvalho N, Gutierrez-Delgado C, et al. Intervention strategies to reduce the burden of non-communicable diseases in Mexico: cost effectiveness analysis. BMJ. 2012;344(mar02 1):e355.

27. Becker SM. Detection of somatization and depression in primary care in Saudi Arabia. Soc Psychiatry Psychiatr Epidemiol. 2004;39:962-6.

28. WHO-CHOICE methods, In: Cost effectiveness and strategic planning (WHO-CHOICE). World Health Organization. https://www.who.int/choice/ cost-effectiveness/methods/en/. Accessed 2 Nov 2019.

29. FutureDocs Forecasting Tool. 2016. https://www2.shepscenter.unc.edu/ workforce/index.php.

30. Memish ZA, Jaber S, Mokdad AH, AlMazroa MA, Murray CJL, Al Rabeeah AA. Burden of disease, injuries, and risk factors in the Kingdom of Saudi Arabia, 1990-2010. Prev Chronic Dis. 2014. https://doi.org/10.5888/ pcd11.140176.

31. Cartmill L, Comans TA, Clark MJ, Ash S, Sheppard L. Using staffing ratios for workforce planning: evidence on nine allied health professions. Hum Resour Health. 2012;10(1):2.

32. United Nations Interagency Task Force on the Prevention and Control of Noncommunicable Diseases. The investment case for noncommunicable disease prevention and control in the Kingdom of Saudi Arabia: return on investment analysis and institutional and context analysis; 2018; Geneva: World Health Organization. 
33. Meo SA. Prevalence and future prediction of type 2 diabetes mellitus in the Kingdom of Saudi Arabia: a systematic review of published studies. J Pak Med Assoc. 2016;66:722-5.

34. Sheikh Jl, Cheema S, Chaabna K, Lowenfels AB, Mamtani R. Capacity building in health care professions within the Gulf cooperation council countries: paving the way forward. BMC Med Educ. 2019;19(1):83.

35. El-Jardali F, Jamal D, Abdallah A, Kassak K. Human resources for health planning and management in the Eastern Mediterranean region: facts, gaps and forward thinking for research and policy. Hum Resour Health. 2007;5(1):9.

36. El-Jardali F, Murray S, AbuAIRub R, Dumit N, Al Surimi K, Clinton M, Jama D, Jaafar M. Exploring the problem of scarcity of nurses in underserved areas in the Middle East: factors, reasons and incentives for recruitment and retention. In: Research Report Submitted to Alliance for Health Policy and Systems Research 2012.

37. Alshaikh MK, Filippidis FT, Al-Omar HA, Rawaf S, Majeed A, Salmasi AM. The ticking time bomb in lifestyle-related diseases among women in the Gulf Cooperation Council countries; review of systematic reviews. BMC Public Health. 2017;17(1):536.
38. Alhyas L, McKay A, Majeed A. Prevalence of type 2 diabetes in the States of the co-operation council for the Arab States of the Gulf: a systematic review. PLoS ONE. 2012;7(8):e40948.

39. Rahim HF, Sibai A, Khader Y, Hwalla N, Fadhil I, Alsiyabi H, Mataria A, Mendis S, Mokdad AH, Husseini A. Non-communicable diseases in the Arab world. Lancet. 2014;383(9914):356-67.

40. Ng SW, Zaghloul S, Ali HI, Harrison G, Popkin BM. The prevalence and trends of overweight, obesity and nutrition-related non-communicable diseases in the Arabian Gulf States. Obes Rev. 2011;12(1):1-3.

41. Campbell J, Dussault G, Buchan J, Pozo-Martin F, Guerra Arias C, Leone A, Siyam A, Cometto G. A universal truth: no health without a workforce. Global Health Workforce Alliance and WHO, Geneva. 2013.

\section{Publisher's Note}

Springer Nature remains neutral with regard to jurisdictional claims in published maps and institutional affiliations.
Ready to submit your research? Choose BMC and benefit from:

- fast, convenient online submission

- thorough peer review by experienced researchers in your field

- rapid publication on acceptance

- support for research data, including large and complex data types

- gold Open Access which fosters wider collaboration and increased citations

- maximum visibility for your research: over 100M website views per year

At BMC, research is always in progress.

Learn more biomedcentral.com/submissions 